\title{
Price sensitivity to tourism activities: looking for determinant factors
}

\author{
LORENZO MASIERO
}

Institute for Economic Research (IRE), Faculty of Economics, University of Lugano, Via Maderno 24, CP 4361, CH-6904 Lugano, Switzerland. E-mail: lorenzo.masiero@usi.ch. (Corresponding author.)

\author{
JUAN L. NiCOLAU
}

Department of Financial Economics, Accounting and Marketing, Faculty of Economics, University of Alicante, Spain. E-mail: jl.nicolau@ua.es.

\begin{abstract}
The literature contains evidence that there is a marked heterogeneity in price responses to tourism products, leading to a great variety of tourist sensitivities to price. Thus the role price plays is complex, and a particularly challenging aspect of this complexity is that its effect is not unambiguous, thereby negating the idea that the demand for tourism products and tourist activities can always be regarded as demand for ordinary goods. This article identifies and explains, as a novelty for the tourism industry, price sensitivities to tourism activities individual by individual. The operative formalization uses a mixed logit model to estimate the individual sensitivities to price, and then a regression analysis is applied to detect their determinants. The empirical application finds that motivations, influenced by age, and length of stay with a non-linear effect, are explanatory factors of tourists' price sensitivity to activities.
\end{abstract}

Keywords: tourism activities; response to price; price sensitivity; market heterogeneity; tourist choice

The variable price is a central element in the tourist market, since it is a flexible tool that permits rapid changes (in response to a rival's action) and a powerful competitive element (as individuals can make direct comparisons among different alternatives). Consequently, knowing the impact of prices on individuals' choices is central for tourism economics and management.

However, in a markedly heterogeneous market, like the tourist market, there is an enormous diversity of sensitivities to price. If managers knew the individual-by-individual preference structure in terms of price sensitivities, they would be able to adapt each product to each person. Although knowledge of this price preference structure would be valuable, the main limitation of analysing this internal dimension is that it is not easy to measure on an individual basis, as it is not directly observable. 
In light of the above, the objective of this study is to characterize, as a novelty in the tourism industry, tourists' price sensitivities to tourism activities, individual by individual, by taking into consideration heterogeneity. For this purpose, the article proposes a mixed logit model to find the heterogeneity between individual preferences and to estimate individual responses to price. A regression analysis is then applied to detect the factors that explain these heterogeneous responses.

The article is organized as follows. The next section reviews the role of price in tourism and states hypotheses on the effects of the proposed explanatory variables. The subsequent section covers the design of the investigation, describing the methodology, sample and variables. In the penultimate section we present and discuss the results. Finally, the conclusions are summarized.

\section{The role of price in tourism}

Classical economic theory provides guidelines as to the nature of the demandprice relationship, accepting that price and demand are inversely related. However, at this point it is important to remember the now famous vexatiousness with which Crouch (1994) describes the role of prices in tourism. Certainly, the analysis of prices is one of the trickiest issues to deal with in tourism economics - remember, for example, not only the usual strategy of keeping prices low to be more attractive (Mangion et al, 2005) but the upward movements in prices to improve financial returns (Moriarty, 2010), as well as the market power and negotiating ability of intermediaries to influence prices (Aguiló et al, 2003). Price decisions are also among the more arduous tasks in marketing (Cooper et al, 2008). In this regard, there is considerable evidence from both marketing and economics that price is a complex construct that is multidimensional in nature, especially because of the duality in the effect of price: price can be an index of the amount of sacrifice the individual has to incur to consume the product, as well as of the level of quality that the individual might expect (Dodds et al, 1991; Murphy and Pritchard, 1997). In fact, Dodds and Monroe (1985) show that this dual effect will affect people's predisposition to buy.

The consumer search for quality is particularly intense in tourism not only because of quality in its own right but also because of the uncertainty reduction expected from it. On account of the perishability, inseparability, intangibility and heterogeneity inherent in tourism products, an individual may rely on prices to diminish uncertainty and make sure of what she or he will obtain. Remember that attributes of tourism products are difficult to grasp in advance, as their values are not observed before the service encounter (Espinet et al, 2003). Tourists value the services included in a package that differentiate it, but they do this evaluation by looking at prices (Aguiló et al, 2001). Certainly, prior to consumption, the individual forms expectations about the forthcoming experience using a number of intrinsic and extrinsic cues that indicate the likely performance standards (Gould-Williams, 1999). In this context, price is a quality-extrinsic signalling element (Zeithaml, 1988). And all this is without considering the underlying hedonistic character often found in the consumption of tourism products through which high prices do not always act against demand (Morrison, 1996). 


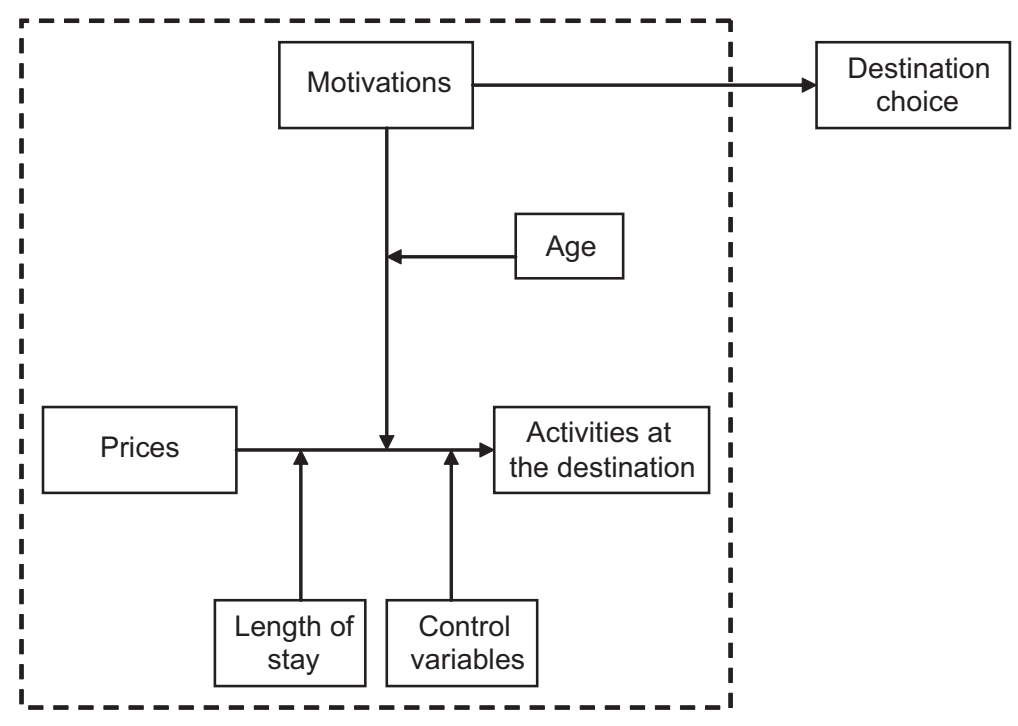

Figure 1. Determinants of individual sensitivities to price of tourism activities.

Therefore, in the context of tourism with a strong response heterogeneity and a great variety of sensitivities to price, the role that price plays becomes especially complex and its effect is not unambiguous - thus dismissing the idea that, in all cases, the demand for tourism products and tourist activities is that of ordinary goods in such a way that price increments diminish consumption. In this context, therefore, price cannot be universally regarded as a factor that reduces the utility of a destination. Consequently, the different price effects and, therefore, these distinct sensitivities to price should implicitly include several factors that explain this heterogeneity.

In this analysis, we focus on an area of research that has not been widely studied previously: the identification and explanation of individual price sensitivities to tourism activities. In the next section, we present the relationships between several factors and these sensitivities.

\section{Determinants of the individual response to prices of tourism activities}

We propose several factors that may explain the individual price sensitivities to tourism activities and outline their effects (see the dashed-line box in Figure 1). We expect the motivations that lead to the selection of a destination to have an effect on the predisposition to pay for the activities available at the destination; an effect that can be influenced by age. Also, as the number of days the individuals spend at the destination determines their level of expenditure, the length of stay should have an impact on price sensitivity to tourism activities. For each relationship, justification is provided below.

Motivations. Tourist motivations lead to the choice of a destination (Lo and Lee, 2010), and this relationship between destination choice and motivation is relevant in identifying the maximum price tourists are prepared to pay. The 
theory of consumer behaviour considers that motivations represent individual internal forces that lead to action (Schiffman and Kanuk, 2007), and in this respect, tourist motivations act as push factors leading to the realization of tourist travel (Sirakaya et al, 1996; Kim and Lee, 2002). As the selection of a certain holiday destination implies a desire for some kind of benefit (such as participation in a given activity), motivations are essentially the reasons why people take a holiday (Richards, 2002). This fact can have an influence on people's predisposition to pay to take part in an activity. According to Rugg's (1973) proposal, a stay in a destination for a period enables enjoyment of the attributes of the destination from which tourists obtain utility. As far as the set of activities offered by a destination can be considered an attribute that describes it, tourists may be prepared to pay extra to obtain utility from participating in specific activities. This last statement applies as long as those activities are in line with people's motivations; otherwise, the reverse would apply - motivations that are not in line with the activities would make people more reluctant to pay extra. Bearing in mind these considerations, we expect that motivations affect people's price sensitivity to activities, and the following hypothesis is stated:

H1: Motivations to go to a destination affect the individual's price sensitivity to activities.

Although motivations have proven to have an effect on tourist decisions, their effect can be influenced by age. Age is a dimension that generates different points of view towards leisure and tourism consumption (Opaschowski, 1990); for example, young and middle-aged people consider holidays as part of their lifestyle, and accept that extra holiday spending has to be saved for during the rest of the year. In fact, a tendency has been detected among young people to prioritize holiday expenditure, although, once they are at the chosen destination, half of these holidaymakers restrict their spending (BAT, 1989). This finding implies that the above hypothesis about the effects of motivations on an individual's price sensitivity to activities may be different depending on the person's age. In fact, this could explain the suggestion in the literature that the assumption of a linear relationship between age and vacation travel decisions seems excessively simplistic and unrepresentative of the real behaviour of individuals (Walsh et al, 1992; Eymann and Ronning, 1992, 1997). That is, the same motivation can exert a different influence on a specific decision contingent on people's age. Therefore, Hypothesis 1 is qualified by Hypothesis 2 as follows:

H2: The effect of motivations to go to a destination on the individual's price sensitivity to activities is influenced by the age of the tourists.

Duration of stay. As the number of days a tourist spends away is 'holiday quantity' (Silberman, 1985), a positive relationship between the duration of stay and expenditure incurred during the holiday is found in the empirical literature: that is, a greater number of days implies greater expenditure (Spotts and Mahoney, 1991; Taylor et al, 1993; Nogawa et al, 1996; Seaton and Palmer, 1997; Van Limburg, 1997; Leones et al, 1998; Mules, 1998; Agarwal and 
Yochum, 1999; Aguiló and Juaneda, 2000; Cannon and Ford, 2002). However, as more days lead to more expenses, it is expected that the tourist will take extra care when it comes to choosing higher-priced or lower-priced activities. That is, assuming two individuals, each with the same budget, but one with a length of stay double that of the other (say, ten days versus five), then when selecting an activity the first will pay greater attention to its price as his or her budget has to be spread over ten days rather than five. Consequently, we propose the following hypothesis:

\section{H3: A longer length of stay leads to higher price sensitivity to activities.}

As before, a nuance has to be taken into account when analysing the effect of the duration of stay on price sensitivity to activities. In particular, doubts can be cast on a linear relationship throughout the range of this continuous variable. Although longer stays are hypothesized to bring about higher price sensitivity to activities, there is no reason to assume a linear effect; quite the contrary, on account of the type of accommodation, tourists' sensitivities may change. Staying in a hotel, generally associated with shorter stays and where a large proportion of the tourist's budget goes to pay for the hotel, is not the same as staying in a second home, where not only can you stay longer with lower costs per person per night, but you also have a lot more discretionary money available (Agarwal and Yochum, 1999). Consequently, considering that we are dealing with a continuous variable, we propose the following hypothesis:

\section{H4: The effect of the length of stay on price sensitivity to activities is non-linear.}

Finally, we also consider the tourist's country of origin as a control variable to observe potential differences in average price sensitivities to activities.

\section{Research design}

\section{Sample, data and variables}

The data used for the analysis refers to a stated choice experiment ${ }^{1}$ conducted in the Ticino region of Switzerland during summer 2010 as part of a project designed to investigate the preferences of tourists for different activities at the destination and the potential for a regional tourist card.

The design of the choice experiment is based on four attributes identified by applying a principal component analysis on the activities undertaken by tourists $^{2}$ - culture and nature, entertainment and sport, evening activities and water activities, respectively. The daily price was added as a fifth attribute, whereas free access to public transport (commonly included in tourist cards) was made explicit in each hypothetical situation generated by the experimental design. For each attribute associated with activities at the destination, three levels were defined, taking into consideration the factor loading of the activities undertaken by tourists. Concerning the price attribute, an exploratory analysis on the tourist cards present in the real market was carried out in order to define the attribute levels to be used in the experiment: 30, 45 and $60 \mathrm{CHF}$, 
Table 1. Attributes and their levels.

Attribute

Culture and nature

Entertainment and sport

Evening activities

Water activities

Price

\section{Attribute levels}

Free usage of cable cars

Free entrance to museums

Free entrance to botanical gardens

Free entrance to entertainment parks

$20 \%$ discount on wellness facilities

$20 \%$ discount on sports and renting sport equipment

$20 \%$ discount on events and festivals

$20 \%$ discount in restaurants

$20 \%$ discount in bars/clubs

Free boat trips on the lake

Free entrance to Lido

$20 \%$ discount on renting a boat

$30 \mathrm{CHF} /$ day

$45 \mathrm{CHF} /$ day

$60 \mathrm{CHF} /$ day

respectively. ${ }^{3}$ Table 1 describes the attributes and the attribute levels considered in the stated choice experiment.

The stated choice experiment was conducted by face-to-face interviews in which tourists were asked to make two choices, first choosing card A, card B, or neither card and, second, choosing between card A or card B. Each respondent was presented with 12 choice situations ${ }^{4}$ (see Figure 2 for an example of a choice card). The dataset analysed in this study refers to the first choice dataset - of card A, card B, or neither.

Nine main locations in Ticino were identified for conducting the survey with tourists, taking into consideration both the most touristic places and the geographical dimension. The sample was comprised of 261 tourists and the survey resulted in 3,132 valid choice observations.

The descriptive statistics for the main socio-demographic and socio-economic groups are reported in Table 2. Within the sample, $70 \%$ of the tourists are between 31 and 60 years old, $10 \%$ are older than 61 , and $5 \%$ are under 20 . With regard to nationality, the most represented are Swiss, followed by Germans and Italians. This pattern reproduces correctly the market shares experienced in Ticino for the three main countries driving tourism demand. Regarding length of the stay and daily budget, the sample interviewed stayed on average for 4.8 nights in Ticino and had an average daily budget of $65 \mathrm{CHF}$ for activities at the destination.

Along with the stated choice experiment, the tourists interviewed were also asked to state their degree of agreement with a set of motivations that had been relevant to their choice of Ticino as holiday destination. In particular, we focus on seven statements included in the survey and measured according to a fourpoint Likert scale - not at all important (1), rather unimportant (2), rather important (3) and very important (4), respectively. 


\begin{tabular}{|c|c|c|c|}
\hline & Card A & Card B & None \\
\hline Public transport & Free public transport in Ticino & Free public transport in Ticino & \\
\hline Culture and nature & Free entrance to museums & Free entrance to botanical parks & \\
\hline Entertainment and sport & $20 \%$ discount on wellness facilities & $\begin{array}{l}20 \% \text { discount on sports and renting } \\
\text { sport equipment }\end{array}$ & \\
\hline Evening activities & $20 \%$ discount in restaurants & $20 \%$ discount in bars/clubs & \\
\hline Water activities & Free entrance to lido & Free boat trips on the lakes & \\
\hline Price & $45 \mathrm{CHF}$ & $30 \mathrm{CHF}$ & \\
\hline \multicolumn{4}{|l|}{ Which card would you buy? } \\
\hline $\begin{array}{l}\text { If you could only chose } \\
\text { between A and B, which one } \\
\text { would you prefer? }\end{array}$ & & & \\
\hline
\end{tabular}

Figure 2. Example of a choice card.

Table 2. Sample descriptive statistics.

Variable

Gender

Age

Country

Average overnight stay

Average daily budget

\section{Sample}

Females (60\%); males (40\%)

20 or under (5\%); $21-30(16 \%) ; 31-40(20 \%)$; $41-50(25 \%) ; 51-60(24 \%) ; 61-70(9 \%) ; 71$ or older $(1 \%)$

Switzerland (45\%); Germany (17\%); Italy (16\%); other $(22 \%)$

4.8 nights

$65 \mathrm{CHF}$

Table 3 illustrates the sample mean and median for the seven motivations considered in this study. According to the sample surveyed, 'getting rest and relaxation' and 'going to a sunny place' are perceived as very important motivations in the decision process for selecting Ticino as holiday destination, as shown by the high value of the sample median. This result is in line with the image that tourists commonly associate with Ticino. Indeed, tourism in Ticino is active mainly during the warm seasons, spring and summer. Two other motivations had played an important role in the selection of the destination: 'trying new food' and 'visiting historical places', which registered a sample median of 3 
Table 3. Sample descriptions for motivations.

\begin{tabular}{lcc}
\hline Motivation & Mean & Median \\
Trying new food & 2.6 & 3 \\
Visiting historical places & 2.8 & 3 \\
Experiencing a simpler lifestyle & 2.2 & 2 \\
Feeling safe and secure & 2.4 & 2 \\
Feeling at home away from home & 2.3 & 2 \\
Going to a sunny place & 3.4 & 4 \\
Getting rest and relaxation & 3.5 & 4 \\
\hline
\end{tabular}

('rather important'). On the other side, three of the seven motivations considered - 'experiencing a simpler lifestyle', 'feeling safe and secure' and 'feeling at home away from home' - had exerted a lower degree of influence in the selection of the destination.

\section{Method}

Within a random utility model framework (McFadden, 1974), the utility function, associated with respondent $n$ for alternative $j$ in choice situation $s$, is typically assumed to be linear in parameters, and represented by Equation (1):

$$
U_{n j s}=\alpha_{j}+\sum_{k=1}^{K} \beta_{n k} x_{n j s k}+\varepsilon_{n j s}
$$

where, $\varepsilon_{n j s}$ is the random term that is independent and identically distributed (IID) extreme value type 1 .

In order to capture the heterogeneity between individual preferences, we rely on the mixed logit class of models ${ }^{5}$ which allow the estimation of both the mean and the standard deviation for the random coefficients according to a specific density function, typically a normal distribution, as follows:

$$
\beta_{n k}=\beta_{k}+\sigma_{k} v_{n k}
$$

where $\beta_{k}$ is the sample mean, $v_{n k}$ is the individual specific heterogeneity with mean zero and standard deviation one, and $\sigma_{k}$ is the standard deviation of $\beta_{n k}$ around $\beta_{k}$, which in our case follows a normal distribution.

Under these assumptions, the probability that respondent $n$ chooses alternative $j$ is described as follows:

$$
P_{n}=\int\left(\Pi_{s} \frac{\exp \left(\alpha_{i}+\sum_{k} \beta_{n k} x_{n i s k}\right)}{\sum_{j} \exp \left(\alpha_{j}+\sum_{k} \beta_{n k} x_{n j k k}\right)}\right) f(\beta) d(\beta)
$$

where $s=1, \ldots, S$ represents the choice situations. Since the integral does not have a closed form, the estimation of the utility parameters is derived from the maximization of the following simulated log likelihood: 


$$
L_{n}=\Sigma_{n} \ln \frac{1}{R} \Sigma_{r} \Pi_{s} \frac{\exp \left(\alpha_{i}+\sum_{k} \beta_{n k} x_{n i s k}\right)}{\sum_{j} \exp \left(\alpha_{j}+\sum_{k} \beta_{n k} x_{n j s k}\right)}
$$

where $r=1, \ldots, R$ refers to the number of draws used for the identification of each random parameter. In this article, we refer to 500 Halton draws. ${ }^{6}$

The individual specific estimates for the price coefficient are then computed by using the Bayes Identity:

$$
g\left(\beta \mid y_{n}\right) \operatorname{Prob}\left(y_{n}\right)=\operatorname{Prob}\left(y_{n} \mid \beta\right) f(\beta)
$$

where $y_{n}$ indicates the alternative $j$ chosen by respondent $n$. The Bayes Identity in (5) allows us to formulate the conditional density of $\beta_{n}$ :

$$
g\left(\beta_{n} \mid y_{n}\right)=\frac{L_{n}\left(y_{n} \mid \beta\right) f(\beta)}{P_{n}}
$$

From the conditional density in (6) we can obtain the conditional expectation as follows:

$$
E\left(\beta_{n} \mid y_{n}\right)=\int \beta g\left(\beta \mid y_{n}\right) d \beta=\int \frac{\beta \operatorname{Prob}\left(y_{n} \mid \beta\right) g\left(\beta \mid y_{n}\right) d \beta}{P_{n}}
$$

Given that the integral in the conditional mean for $\beta_{n}$ does not exist in closed form, the individual specific estimates for the price coefficient are then simulated as:

$$
E\left(\beta_{n} \mid y_{n}\right)=\frac{1}{R} \sum_{r} \beta_{n r} \omega_{n r} \quad \text { where, } \omega_{n r}=\frac{L_{n}}{\sum_{r} L_{n}}
$$

In order to investigate the determinant factors for the heterogeneity in the price sensitivity, a regression is then performed on the estimated individual coefficients associated with the price attribute in the stated choice experiment. Formally, the OLS regression takes the following specification:

$$
\beta_{n}=\alpha+\Sigma_{k} \delta_{k} x_{n k}+u_{n}
$$

where, $\alpha$ is the constant, $\delta_{k}$ the estimated parameter associated with individual specific variable $x_{k}$ and $u_{n}$ is the error term that is normally distributed with mean zero and standard deviation $\sigma$.

\section{Results}

We first estimate the stated choice experiment using a mixed logit model, in which the coefficient of interest - price sensitivity - is included and gauged. 
Table 4. Stated choice model of tourism activities.

Coefficient $\quad t$-ratio

Means for random and non-random parameters

ASC Alt A

2.5405

$15.03^{\mathrm{a}}$

ASC Alt B

2.4058

$14.23^{\mathrm{a}}$

Price

$-0.0398$

$-9.58^{\mathrm{a}}$

Cable car

$-0.0742$

$-1.45$

Museum

Entertainment park

0.2183

$3.41^{\mathrm{a}}$

$-0.0714$

$-1.42$

$20 \%$ discount on sport and renting sports equipment

$-0.0309$

$-0.56$

$20 \%$ discount on restaurants and bars

$-0.2456$

$-2.70^{\mathrm{a}}$

0.3516

$6.77^{\mathrm{a}}$

Free boat trips on the lake

$-0.2484$

$-3.76^{a}$

Standard deviations for random parameters

Ns Price

0.0410

$14.73^{\mathrm{a}}$

Ns Cable car

0.1434

0.4724

$7.90^{\mathrm{a}}$

Ns Entertainment park

0.0057

0.03

Ns $20 \%$ discount on sport and renting sports equipment

0.2059

$2.22^{\mathrm{b}}$

Ns $20 \%$ discount on restaurants and bars

0.6380

$5.83^{\mathrm{a}}$

Ns Free boat trips on the lake

0.3991

$6.86^{\mathrm{a}}$

Ns 20\% discount on renting a boat

0.1462

Sample

Halton draws

Restricted LL

Final log-likelihood

Number of parameters

AIC

McFadden pseudo $\rho^{2}$

0.1390

Note: ${ }^{\mathrm{a}}$ prob $<1 \%$; ${ }^{\mathrm{b}}$ prob $<5 \%$; ${ }^{\mathrm{c}}$ prob $<10 \%$.

Table 4 shows the global results that represent the preferences of an average tourist. We find that price is significant at a level below 0.001, and presents a negative sign. This leads us to characterize it as dissuasive factor in the choice of activities. However, it is important to stress that the standard deviation parameter of the coefficient is significant, which implies that 'price' has a differentiated effect among the individuals of the sample and, thus, a given high price does not suppose the same reduction in utility for all the tourists sampled. The differentiated effect found for 'price' suggests that there is a great diversity of sensitivities in the tourist market.

Once the individual sensitivities to price have been estimated, they are used as the dependent variable of a regression analysis so as to detect its explanatory factors and test the hypotheses stated (Table 5). Note that, even though the $R$-square is not as good as desirable, it is important to observe that the regression is globally significant $(\mathrm{F}=2.3 ; p<0.01)$, and, most importantly, 16 coefficients are significant out of 20 . This means that the proposed variables 
Table 5. Determinant factors of price sensitivity to activities.

Coefficient

$$
0.0164
$$

$-0.0116$

$-0.0136$

0.0145

$-0.0125$

$-0.0134$

0.0202

$-0.0032$

0.0043

0.0035

$-0.0036$

0.0027

0.0028

$-0.0059$

$-0.0019$

0.0003

$-0.0161$

$-0.0064$

0.0043

$-0.0356$ t-ratio

$2.25^{\mathrm{b}}$

$-1.78^{\mathrm{c}}$

$-1.74^{\mathrm{c}}$

$2.09^{\mathrm{b}}$

$-1.85^{c}$

$-1.71^{\mathrm{c}}$

$2.26^{\mathrm{b}}$

$-1.83^{\mathrm{c}}$

$2.72^{\mathrm{a}}$

$1.87^{\mathrm{c}}$

$-2.11^{\mathrm{b}}$

1.58

1.47

$-2.87^{\mathrm{a}}$

$-2.14^{\mathrm{b}}$

$1.71^{\mathrm{c}}$

$-2.14^{\mathrm{b}}$

$-0.87$

0.73

$-2.28$

Constant

Sample

Number of parameters

$R$-squared

Adjusted $R$-squared

0.088

Note: $\mathrm{a}=$ prob $<1 \% ; \mathrm{b}=$ prob $<5 \% ; \mathrm{c}=$ prob $<10 \%$.

are clearly determinant factors of this internal aspect of the individual and that further research is needed to find more elements that explain price sensitivity in the context of tourism activities - thereby opening up new threads of research. Turning to the specific results, we find the following.

As for motivations, we obtain significant parameters for the seven motivations, supporting Hypothesis 1 that motivations to go to a destination reduce the individual's price sensitivity to activities. In particular, positive parameters are found for 'trying new food' $(p<0.025)$, 'feeling safe and secure' $(p<0.037)$ and 'getting rest and relaxation' $(\phi<0.024)$, meaning that people with these motivations when selecting a destination are less affected by higher prices when choosing activities. On the other hand, we find negative parameters for 'visiting historical places' $(p<0.075)$, 'experiencing a simpler lifestyle' $(\phi<0.082)$, 'feeling at home away from home' $(p<0.064)$ and 'going to a sunny place' $(p<0.088)$. One can assume that most tourists are not prepared to pay extra for activities that allow them to experience a simpler lifestyle, feel at home away from home or go to a sunny place merely for the sun: people with these motivations should obtain their sought benefits without incurring greater costs. However, it is surprising to see that people with the motivation 'visiting historical places' become more sensitive to price, which in principle is against 
expectations. Nevertheless, this result is still reasonable, as the cultural activities proposed in the cards were free in all cases. Thus when culturemotivated people select the activities, once they have made sure that they will get what they expect at the destination (visiting historical places), they do not much care about the non-cultural activities and will go for the cheaper option.

Regarding interactions, five out of seven interactions are significant: as people get older, the positive effects of 'trying new food' $(p<0.068)$, 'feeling safe and secure' $(p<0.035)$ and 'getting rest and relaxation' $(p<0.004)$ on price sensitivity to activities are reduced; and the negative effects of 'visiting historical places' $(p<0.006)$ and 'experiencing a simpler lifestyle' $(\phi<0.061)$ diminish too. These significant interactions confirm Hypothesis 2 that the effect of motivations to go to a destination on the individual's price sensitivity to activities is influenced by age.

Concerning duration of stay, we find a negative parameter for the variable 'number of nights' $(p<0.032)$ and a positive parameter for its squared value $(p<0.088)$. These results support Hypothesis 3 that longer lengths of stay lead to higher price sensitivity to activities, as the budget has to be divided by more days, and Hypothesis 4 that the effect of length of stay on price sensitivity to activities is non-linear. In particular, 'the longer stays, the higher sensitivity' statement holds up to a point (specifically, up to day five); after that, for stays of six days or longer, people become less sensitive to prices. As longer durations are enjoyed mostly by people with second homes, on account of the lower costs per person per night, the idea that they have more discretionary money at their disposal can aptly apply, in line with Agarwal and Yochum (1999).

As for the control variable 'tourist's country of origin', we observe differences in sensitivities; in particular, we find that Italians are more reluctant to pay extra money $(p<0.033)$ than any other nationality in the sample.

\section{Conclusions}

Prices in tourism are especially complex and their effects are not unambiguous, characterized by a strong response heterogeneity which leads to a great variety of sensitivities to prices. This implies that, rather than prices being dissuasive elements that diminish utility, they can sometimes have positive - or, to be more precise, less negative - effects.

In this vein, the objective of this study is to analyse, for the first time, tourists' price sensitivities to tourism activities, individual by individual, by taking into consideration heterogeneity. To do this, we use a mixed logit model to find the heterogeneity between individual preferences and estimate the individual responses to price, and then apply a regression analysis to identify factors that explain these heterogeneous responses.

The results of the empirical application show that the demand for tourist activities is that of ordinary goods insofar as price increases diminish consumption; however, 'price' has a differentiated effect among individuals, and thus a particular high price does not have the same reduction in utility for all tourists. Specifically, we find that motivations exert an effect on price sensitivity to activities, some diminishing and others increasing it. At this point, it is important to stress that the effect of motivations is influenced by the tourist's 
age, such that the same motivation might impact differently on an individual's price sensitivity depending on his or her age. Concerning length of stay, we find that longer stays lead to higher price sensitivity to activities (because of people's limited budget), but there is a threshold in day five; from day six, people become less sensitive (as these longer durations are mainly associated with people who have a second home and therefore have more money for discretionary expenditure).

With regard to the implications for management, the first point to note is that a knowledge of the determinant factors of price sensitivities to activities allows destinations and travel organizations to improve the effectiveness of their price policies and strategies by taking into account those characteristics of the selected target group that will lead them to accept a price to a greater or lesser extent. Given the confirmation of the existence of a diversity of price sensitivities, a critical implication is that knowing the tourist by a tourist preference structure in terms of price sensitivities enables more tailored pricing strategies. It is important to remember that the analysis of 'price preferences' requires looking into an internal dimension which is not directly observable.

Further avenues of research remain to be explored. First, more explanatory factors of this internal aspect of the individual's price sensitivity to tourism activities are needed. Second, a posteriori analysis could help to identify segments according to their price sensitivities to activities, which would definitely allow organizations to detect their target groups.

\section{Endnotes}

1. Stated choice experiments, also known as stated preference experiments, are statistical experiments involving hypothetical choice situations and predefined (by the researcher) attributes and attribute levels (see Louviere et al, 2000 for details).

2. The entire survey involved two waves. Data collected from the first wave were used to identify the four attributes (via principal component analysis) characterizing the stated choice experiment conducted in the second wave.

3. Approximate exchange rate at December 2010: $1 \mathrm{CHF}=1.03$ US $\$$.

4. The experimental design refers to an orthogonal design performed by Ngene (www.choicemetrics.com). The final design resulted in two blocks of twelve choice situations each.

5. See Hensher and Greene (2003) for a detailed discussion on mixed logit models.

6. See Train (2009) for details about Halton draws.

\section{References}

Agarwal, V.B., and Yochum, G.R. (1999), 'Tourist spending and race of visitors', Journal of Travel Research, Vol 38, pp 173-176.

Aguiló, E., and Juaneda, C. (2000), 'Tourism expenditure for mass tourism markets', Annals of Tourism Research, Vol 27, No 3, pp 624-637.

Aguiló, E., Alegre, J., and Sard, M. (2003), 'Examining the market structure of the German and UK tour operating industries through an analysis of package holiday prices', Tourism Economics, Vol 9, No 3, pp 255-278.

Aguiló, P.M., Alegre, J., and Riera, A. (2001), 'Determinants of the price of German tourist packages on the island of Mallorca', Tourism Economics, Vol 7, No 1, pp 59-74.

BAT Freizeitforschungsinstitut (1989), Wie arbeiten wir nach dem Jahr 2000?, Freizeit-Impulse für die Arbeitswelt von Morgen, Hamburg.

Cannon, T.F., and Ford, J. (2002), 'Relationship of demographic and trip characteristics to visitor spending: an analysis of sports travel visitors across time', Tourism Economics, Vol 8, No 3, pp 263271. 
Cooper, C., Fletcher, J., Fyall, A., Gilbert, D., and Wanhill, S. (2008), Tourism: Principles and Practice, Financial Times Prentice Hall, Harlow.

Crouch, G.I. (1994), 'The study of international tourism demand: a review of findings', Journal of Travel Research, Vol 33, pp 12-23.

Dodds, W.B., and Monroe, K.B. (1985), 'The effect of brand and price information on subjective product evaluations', Advances in Consumer Research, Vol 12, pp 85-90.

Dodds, W.B., Monroe, K.B., and Grewal, D. (1991), 'Effects of price, brand, and store information on buyers' product evaluations', Journal of Marketing Research, Vol 28, No 3, pp 307-319.

Espinet, J.M., Saez, M., Coenders, G., and Fluvià, M. (2003), 'Effect on prices of the attributes of holiday hotels: a hedonic prices approach', Tourism Economics, Vol 9, No 2, pp 165-177.

Eymann, A., and Ronning, G. (1992), 'Discrete choice analysis of foreign travel demand', in Vosgerau, H.J., ed, European Integration in the World Economy: Studies in International Economics and Institutions, Springer, Berlin.

Eymann, A., and Ronning, G. (1997), 'Microeconometric models of tourists' destination choice', Regional Science and Urban Economics, Vol 27, pp 735-761.

Gould-Williams, J. (1999), 'The impact of employee performance cues on guest loyalty, perceived value and service quality', Service Industries Journal, Vol 19, No 3, pp 97-118.

Hensher, D.A., Greene, W.H. (2003), 'Mixed logit models: state of practice', Transportation, Vol 30, No 2, pp 133-176.

Kim, S., and Lee, Ch. (2002), 'Push and pull relationships', Annals of Tourism Research, Vol 29, No 1, pp 257-260.

Leones, J., Colby, B., and Crandall, K. (1998), 'Tracking expenditures of the elusive nature tourists of Southeastern Arizona', Journal of Travel Research, Vol 29, No 3, pp 32-36.

Lo, A.S., and Lee, C.Y.S. (2010), 'Motivations and perceived value of volunteer tourists from Hong Kong', Tourism Management, Vol 32, No 2, pp 326-334.

Louviere, J.J., Hensher, D.A., and Swait, J.F. (2000), Stated Choice Methods: Analysis and Applications, Cambridge University Press, Cambridge.

Mangion, M.L., Durbarry, R., and Sinclair, M.T. (2005), 'Tourism competitiveness: price and quality tourism competitiveness: price and quality', Tourism Economics, Vol 11, No 1, pp 45-68.

McFadden, D. (1974), 'Conditional logit analysis of qualitative choice behavior', in Zarembka, P., ed, Frontiers in Econometrics, Academic Press, New York.

Moriarty, J.P. (2010), 'Have structural issues placed New Zealand's hospitality industry beyond price?', Tourism Economics, Vol 16, No 3, pp 695-713.

Morrison, A.M. (1996), Hospitality and Travel Marketing, Delmar, New York.

Mules, T. (1998), 'Decomposition of Australian tourist expenditure', Tourism Management, Vol 19, No 3, pp 267-271.

Murphy, P.E., and Pritchard, M. (1997), 'Destination price/value perceptions: an examination of origin and seasonal influences', Journal of Travel Research, Vol 35, pp 16-22.

Nogawa, H., Yamaguchi, Y., and Hagi, Y. (1996), 'An empirical research study on Japanese sport tourism in sport-for-all events: case studies of a single-night event and a multiple-night event', Journal of Travel Research, Vol 35, No 2, pp 46-54.

Opaschowski, H.W. (1990), ‘'Turismo de Masas o Turismo a Medida? Límites Económicos, Ecológicos and Psicológicos', Papers de Turisme, No 4, Unidad de Investigación y Desarrollo, Instituto Turístico de Valencia, Valencia, pp 68-80.

Richards, G. (2002), 'Tourism attraction systems: exploring cultural behaviour', Annals of Tourism Research, Vol 29, No 4, pp 1048-1064.

Rugg, D. (1973), 'The choice of journey destination: a theoretical and empirical analysis', Review of Economics and Statistics, Vol 55, No 1, pp 64-72.

Schiffman, L.G., and Kanuk, L.L. (2007), Consumer Behavior, Prentice Hall, Englewood Cliffs, NJ.

Seaton, A.V., and Palmer, C. (1997), 'Understanding VFR tourism behaviour: the first five years of the United Kingdom Tourism Survey', Tourism Mangement, Vol 18, No 6, pp 345355.

Silberman, J. (1985), 'A demand function for length of stay: the evidence from Virginia Beach', Journal of Travel Research, Vol 23, No 4, pp 16-23.

Sirakaya, E., McLellan, R.W., and Uysal, M. (1996), 'Modeling vacation destinations decisions: a behavioural approach', Journal of Travel E Tourism Marketing, Vol 5, No 1/2, pp 57-75.

Spotts, D.M., and Mahoney, E.M. (1991), 'Segmenting visitors to a destinations region based on the volume of their expenditures', Journal of Travel Research, Vol 29, No 4, pp 24-31. 
Taylor, D.T., Fletcher, R.R., and Clabaugh, T. (1993), 'A comparison of characteristics, regional expenditures, and economic impact of visitors to historical sites with other recreational visitors', Journal of Travel Research, Vol 32, No 1, pp 30-35.

Train, K. (2009), Discrete Choice Methods with Simulation, 2nd edition, Cambridge University Press, Cambridge.

Van Limburg, B. (1997), 'Overnight tourism in Amsterdam 1982-1993: a forecasting approach', Tourism Management, Vol 18, No 7, pp 465-468.

Walsh, R.G., John, K.H., McKean, J.R., and Hof, J.G. (1992), 'Effect of price on forecasts of participation in fish and wildlife recreation: an aggregate demand model', Journal of Leisure Research, Vol 24, No 2, pp 140-156.

Zeithaml, V.A. (1998), 'Consumer perceptions of price, quality, and value: a means-end model and synthesis of evidence', Journal of Marketing, Vol 52, pp 2-22. 